\title{
Comparing two data collection methods to track vital events in maternal and child health via community health workers in rural Nepal
}

Nandini Choudhury

Possible

Aparna Tiwari ( $\nabla$ aparnat19@gmail.com )

Possible https://orcid.org/0000-0003-2333-1833

Wan-Ju Wu

Possible

Ved Bhandari

Nyaya Health Nepal

Laxman Bhatta

Nyaya Health Nepal

Bhawana Bogati

Nyaya Health Nepal

David Citrin

Possible

Scott Halliday

Possible

Sonu Khadka

Nyaya Health Nepal

Nutan Marasini

Nyaya Health Nepal

Sachit Pandey

Nyaya Health Nepal

Madeleine Ballard

Icahn School of Medicine at Mount Sinai Department of Global Health

Hari Jung Rayamazi

Nyaya Health Nepal

Sabitri Sapkota

Possible

Ryan Schwarz

Possible

Lisa Sullivan 
Boston University School of Public Health

\section{Duncan Maru}

Possible

\section{Aradhana Thapa}

Possible

\section{Sheela Maru}

Possible https://orcid.org/0000-0001-8217-3682

\section{Research}

Keywords: vital events, maternal and child health, community health workers, mHealth

Posted Date: May 5th, 2021

DOl: https://doi.org/10.21203/rs.3.rs-482532/v1

License: (c) (i) This work is licensed under a Creative Commons Attribution 4.0 International License. Read Full License 


\section{Abstract}

Background: Timely tracking of health outcomes is difficult in low- and middle-income countries without comprehensive vital registration systems. Community health workers (CHWs) are increasingly collecting vital events data while routinely delivering care in communities in low-resource settings. It is necessary, however, to assess whether routine programmatic data collected by $\mathrm{CHWs}$ are sufficiently reliable for timely monitoring and evaluation of health interventions. To study this, we assessed the consistency of vital events data recorded by CHWs using two methodologies - routine data collected while delivering an integrated maternal and child health intervention, and data from a birth history census approach at the same site in rural Nepal.

Methods: We linked individual records from routine programmatic data from June 2017 to May 2018 with those from census data, both collected by $\mathrm{CHWs}$ at the same site using a mobile platform. We categorized each vital event over a one-year period as "recorded by both methods," "census alone," or "programmatic alone." We further assessed whether vital events data recorded by both methods were classified consistently.

Results: We identified a total of 713 unique births (from maternal records only) and 746 births (collectively from maternal and child records) from June 2017 to May 2018 in one or both data collection methods. About three-fourths ( $n=526)$ of total births (from maternal records only), and ( $n=572)$ of total births (collectively from maternal and child records) were identified by both methods. Among births from maternal records identified by both methods, there was high consistency in birth location classification. Programmatic data captured more births (from mothers and children combined) than census data (723 versus 595). Most infants were consistently classified as "living" in both methods, while infant deaths and stillbirths were largely classified inconsistently or recorded by only one method. Programmatic data identified five infant deaths and five stillbirths not recorded in census data.

Conclusions: Our findings suggest that data collected by CHWs from routinely tracking pregnancies, births, and deaths over time are promising for timely program monitoring and evaluation despite some limitations, and may be more effective than cross-sectional census surveys asking women to recall these events.

\section{Background}

Most low- and middle-income countries (LMICs) rely on national representative sample surveys such as Demographic and Health Surveys (DHS) and Multiple Indicator Cluster Surveys for health outcomes data $(1,2)$. While national surveys like these offer advantages such as national coverage and standardized data collection, they are inadequate for tracking outcomes with higher geographic or temporal resolution $(1,3)$. Timely tracking of vital events and health outcomes is essential for health systems strengthening (4). Such "real-time" data are unavailable, however, in most LMICs in the absence of comprehensive vital registration systems. Facility-based data are often incomplete and unreliable in rural areas for accurately 
calculating community-level metrics since many health events occur outside health facilities and are not captured by facility data systems $(5,6)$.

To accurately and routinely track data at the community level, localized data collection systems are important. In many LMICs, community health workers (CHWs) are increasingly collecting vital events data routinely, including tracking pregnancies, births, and child deaths at the community or village level, as they provide care $(7,8)$. CHWs are recognized as a promising cadre to help achieve universal health coverage in many low-resource settings $(9,10)$. CHWs provide a variety of services in their communities through home-visits, including health screening, counseling, and referrals. Mobile technologies assist CHWs in delivering quality services and collecting data in remote and rural areas (11). With the increase in smartphone users globally, it is increasingly common for $\mathrm{CHW}$ to use mobile technology even in rural settings (12).

Despite the potential of engaging CHWs in timely local data collection and response in LMICs, there have been questions about the quality and completeness of data reported by them for effectively evaluating program and health outcomes. A recent scoping review suggests that community-based vital events reporting is promising but tends to be sub-optimal in data quality and completeness (13). Studies in Mali, Ethiopia, and Malawi, conducted by the 'Real-Time Monitoring of Under-Five Mortality' group, found that vital events data collected routinely by $\mathrm{CHWs}$ varied in quality and completeness to assess mortality rates accurately at scale. CHWs' routine data underreported both births and deaths when compared against household survey estimates $(7,14)$. These studies also highlight the importance of further addressing whether routine data collected by $\mathrm{CHWs}$ are sufficiently reliable for use in priority-setting and decisionmaking, especially since $\mathrm{CHW}$ programs vary widely in supervision, remuneration, and workload (15). Given that many LMICs have a community workforce that can collect and longitudinally track routine vital events data at the household level, it is important to assess whether these "real-time" data collected by $\mathrm{CHWs}$ are reliable for program evaluation.

Like many other LMICs, the vital registration system in Nepal has gaps. For example, only an estimated $42 \%$ of births are recorded (16). It is common to use DHS data, collected every 5 years, to track the progress of population health indicators at a national level (17-19). However, timely tracking of progress in health outcomes at the community level is difficult because of the gaps in localized data collection systems. Municipal level data reporting in Nepal is focused on facility-based indicators and likely misses vital events that take place outside facilities. Nyaya Health Nepal (NHN), a non-governmental organization and its technical partner Possible, attempted to address these gaps through household-level data collection embedded within routine care delivery by a trained, salaried, and supervised cadre of CHWs. This CHW model was part of a pilot study conducted in partnership with the Government of Nepal Ministry of Health and Population in two rural districts in Bagmati Province (Province 3) and Far-Western Province (Province 7). The pilot aimed to study the impact of an integrated intervention delivered by this cadre of $\mathrm{CHWs}$ on reproductive, maternal, newborn, and child health (RMNCH) outcomes (20). CHWs used a mobile platform (CommCare) for counseling, decision support, and simultaneous routine data collection via customized applications and forms $(20,21)$. Upon expanding services in a new catchment 
area, CHWs conducted a one-time household census to identify and enroll married women of reproductive age into the RMNCH care delivery intervention. For each eligible woman, they recorded a birth history for the preceding two years. Following this enrollment period, CHWs regularly visited all enrolled women at home, actively screened for and followed identified pregnancies, and recorded all births in the communities they serve. They also provided postnatal and early childhood care counseling and referrals, monitored the health of under-two children every month, and recorded any child deaths (20).

For the RMNCH pilot study, given resource constraints in the study setting and our goals of improving $\mathrm{CHW}$ data collection and utilization, we decided to use routine programmatic data for monitoring and impact evaluation instead of hiring research enumerators. Using routinely collected data would also facilitate data infrastructure for the $\mathrm{CHW}$ program beyond the study period. To assess whether these routinely collected data by $\mathrm{CHWs}$ were reliable for evaluating health interventions, we designed a smaller study within the broader RMNCH pilot study. We were limited in our resources to assess the completeness of $\mathrm{CHW}$-collected routine programmatic data with the typically used national census methodology or research data enumerators $(22,23)$. Therefore, we sought to assess the consistency of CHW-collected routine programmatic data through comparison with another $\mathrm{CHW}$ data collection method, i.e. conducting a household census with birth histories. We compared each vital event (infant birth, death, or stillbirth) from these two methods to address the following questions: did CHWs identify each birth event by both methods; did CHWs classify the birth location consistently in both methods; did CHWs identify newborns and infants (up to the age of 1 year) by both methods; and did CHWs classify birth and infant outcomes consistently in both methods?

Here, we present our findings from assessing the consistency of CHW-collected maternal and child health vital events using routine programmatic data versus a census birth history, and its implications for using routine data for monitoring and evaluation in a low-resource setting.

\section{Methods}

\section{Study site}

We conducted this study in a population of approximately 36,000 in the Far-Western Province (Province 7). The study site is part of a larger cluster within a non-randomized implementation research trial on CHW-delivered RMNCH care in Provinces 3 and 7 (20). Nepal's hilly Far-Western Province is one of the poorest provinces and was severely affected by the civil war that ended in 2006 (24). NHN has been providing healthcare in this area since 2008, primarily through a hospital run in partnership with the Government of Nepal. In 2016, NHN started its CHW pilot intervention in the hospital's immediate catchment area, which spans fourteen wards and comprises the site for this study. CHWs are local women recruited from the catchment area and have completed a tenth-grade education at minimum. They are full-time, salaried employees who receive pre-service training, structured management support, and direct supervision from community health nurses (CHNs) (20). Each CHW typically serves one ward, an administrative unit of approximately 2,000 people. As part of their responsibilities, CHWs go door-to- 
door to enroll all eligible participants, deliver home-based counseling, conduct basic health assessments, and make referrals for high-risk conditions. CHWs collect data concurrently using customized CommCare smartphone applications with inbuilt forms. These serve both as clinical decision support and data collection tools, allowing CHWs to access and update each participant's longitudinal health record.

From February to July 2016, CHWs attempted to visit each household in the study site to enroll married women of reproductive age into the RMNCH intervention and recorded a three-year birth history for each eligible woman who provided consent. Following this enrollment phase, $\mathrm{CHWs}$ implemented key care delivery components of the RMNCH intervention. These included pregnancy screening and surveillance via quarterly home visits, and monthly antenatal and postnatal care home visits. In February 2017, homebased care was expanded to include monthly $\mathrm{CHW}$ visits for children up to the age of two years. Community health nurses frequently accompanied $\mathrm{CHWs}$ on their home visits for direct supervision.

In 2017, NHN's catchment areas were administratively restructured into rural municipalities as part of a broader, nationwide restructuring of local governance following Nepal's constitutional transition to federalism. During this time, the fourteen wards comprising the study site were reorganized between four distinct municipalities. Following this local restructuring, NHN launched another household census at the study site in July 2018, analogous to the one conducted during the enrollment phase in 2016. The primary purpose of this census was for CHWs to update each household's record using the correct ward and municipality, and update its address and demographic data within the CommCare registry. During this process, CHWs also enrolled any households and eligible women who were not previously enrolled in the RMNCH intervention. Since this household census offered an opportunity for cross-sectional, retrospective data collection, we decided to collect a two-year birth history to compare vital events with those recorded during routine $\mathrm{CHW}$ care delivery.

\section{Study population}

The study population consisted of married women of reproductive age (15-49 years) and their infants (up to one year in age) in fourteen wards. This was the subpopulation of women enrolled in and defined by the larger RMNCH research study, as is standard in interventions focusing on reproductive health. As such, vital events associated with unmarried women or women outside the specified age window were not included. Although the larger RMNCH research study included children up to the age of two years, we included data for infants up to the age of one in this study due to practical constraints.

\section{Data collection}

\section{Routinely collected (programmatic) data}

CHWs recorded vital events data on births (including stillbirths) and deaths as they were identified during routine home visits to deliver RMNCH care. CHWs completed a one-time 'post-delivery form' in CommCare soon after a woman gave birth, comprising programmatic maternal data. They recorded information about the birth location (e.g., hospital, health post, home), birth outcome (stillbirth or live birth), and neonatal deaths among live births (i.e. death within 30 days of birth). Newborn children were enrolled in a separate 'child registry' in CommCare for follow-up care. Of note, children (under two years) could have 
been enrolled in the child registry even if their mothers were not enrolled in care during pregnancy, or if maternal delivery data were unavailable. CHWs then conducted monthly visits to these children and recorded any deaths they identified (Fig. 1). For records with child follow-up data available, we utilized data from the most recent CHW home visit to determine the child's updated status ("living" or "death"). Based on the workflow described above, infant deaths could have been recorded in multiple CommCare forms, including in the mother's 'post-delivery form' and the regularly updated 'child registry'. We extracted data from both forms and merged them to create a composite programmatic record for each mother-child dyad, linking mother's post-delivery data (where available) with each child's individual follow-up care data using a common "household ID" and the birth month and year. We also retained data for children who were enrolled without corresponding maternal data, and vice versa.

\section{Census data}

Our second source of data was the cross-sectional birth history census that CHWs conducted in 2018 while updating household information. NHN's Mobile Systems Engineer created specific forms within CommCare and provided a week-long training for $\mathrm{CHWs}$ and $\mathrm{CHN}$ s to update house numbers in the household registry, and to collect birth history data using a shorter version of the original enrollment census. During household visits, if eligible women agreed to participate, $\mathrm{CHWs}$ recorded their information about each birth in the preceding two years, including birth location, birth outcomes, and infant deaths (Fig. 1).

In addition to ongoing supervision from CHNs, NHN's monitoring and evaluation staff and the research team provided continuous technical support to $\mathrm{CHWs}$ during census data collection, including conducting regular data quality checks. CHWs completed household renumbering in approximately four months between July-October 2018. Pregnancy surveillance (part of the routine RMNCH intervention) was paused during this time to account for the CHWs' additional workload, while other care delivery services continued as usual. Collecting birth histories took $\mathrm{CHWs}$ an additional five months and census data collection ended after March 2019. CHWs noted that the delays were largely because they had to attempt multiple visits for some women who were not at home or had migrated elsewhere when they visited for household renumbering.

\section{Analysis}

Accounting for systematic differences in data collection because of the timing and duration of census data collection described above, we expected both methodologies to theoretically be able to record all vital events in the one year period from June 1, 2017 to May 31, 2018. Birth location was only recorded in maternal data, i.e. in the programmatic 'post-delivery' and census data. Thus, to compare each birth event recorded via maternal data in both sources, we used a unique ID for each eligible woman who gave birth during this period to link and compare records from both sources, counting twins as single births. We then classified each birth as having been recorded in "both methods," "census alone," or "programmatic alone". We categorized each birth location using a binary variable, defining an "institutional" birth as one that took place at any health facility, and a "non-institutional" birth as one that took place at home or on 
the road. We further assessed if births that had been recorded in both data sources were classified consistently as "institutional" or "non-institutional" births in both using cross-tabulations.

To assess child outcomes, we merged programmatic data on births (including stillbirths) and infants from both the routinely collected maternal 'post-delivery form' and 'child registry', with the census birth history using a combination of a family ID and the birth month and year. We used the family ID to merge records since mother-child dyads were not directly linked, and the birth month and year since maternal delivery dates and child birth dates were often off by a few days. In this dataset, we ensured that each birth (collectively from maternal and child records) (including twins) was separated as an individual record to track outcomes. We checked for any duplicates and mismatched records. For any discrepant records identified, we manually verified each data source and cleaned the record, e.g. if a child's date of birth and mother's delivery date differed by a month and resulted in two separate observations for the same birth during the data merge. We then classified each birth/infant in the merged dataset as having been identified in "both methods," "census alone," or "programmatic alone." For births (collectively from maternal and child records) that were identified in both data sources, we cross-tabulated the data to check for consistency in classifying outcomes, i.e. "stillbirth", "living", or "infant death." We also conducted a sensitivity analysis for consistency in birth location and child outcomes classification, in which we included records identified by only one source in addition to those identified in both sources (Supplementary Table 1 and Supplementary Table 2). We conducted all analyses using SAS software version 9.4.

\section{Results}

CHWs identified 6,181 married women of reproductive age during the 2018 census. Of these, 6,100 (99\%) were available at least once during the census data collection period, while 81 (1\%) were never available. Of those available, 5,775 (95\%) agreed to complete the birth history while $325(5 \%)$ declined. During data extraction, there were 1,816 maternal "post-delivery form" records for births in the programmatic data. After limiting these data to births within the one year observation period and removing duplicates, 646 unique birth records from maternal data (counting twins as one record) were retained. We also identified 677 unique records for children born during the observation period and enrolled in follow-up care through programmatic data.

\section{Comparing births (from maternal records only) recorded by both methods}

We identified 713 unique births in maternal records (counting twins as one birth) during the June 1, 2017 May 31, 2018 observation period in one or both data sources. Of these, $526(73.7 \%)$ were identified in both data sources, while 67 (9.3\%) were identified in the census alone, and $120(16.8 \%)$ in the care delivery programmatic data alone (Fig. 2).

Consistency in birth location classification: A total of 513 births (from maternal records only) were categorized consistently as "institutional" or "non-institutional" in both data sources, comprising $97.5 \%$ 
(95\% Cl: $96.2 \%, 98.8 \%$ ) of the 526 birth identified in both sources (Table 1) and 72\% (95\% Cl: $68.7 \%$, $75.3 \%)$ of all 713 births from maternal records.

Table 1

Consistency in location classification for each birth identified in both census and programmatic data, $\mathrm{n}=526$

\begin{tabular}{|lll|}
\hline & \multicolumn{2}{l|}{$\begin{array}{l}\text { Birth location classification in routine } \\
\text { (programmatic) data }\end{array}$} \\
\hline Birth location classification in census data & $\begin{array}{l}\text { Non-institutional births } \\
\mathbf{n ( \% )}\end{array}$ & $\begin{array}{l}\text { Institutional births } \\
\mathbf{n}(\%)\end{array}$ \\
\hline Non-institutional births, $\mathbf{n}(\%)$ & $9(1.7 \%)$ & $2(3.8 \%)$ \\
\hline Institutional births, $\mathbf{n}(\%)$ & $11(2.1 \%)$ & $504(95.8 \%)$ \\
\hline
\end{tabular}

\section{Comparing birth identification in both methods}

We identified 746 unique births (collectively from maternal and child records) between June 1, 2017 and May 31, 2018 in one or both data sources, when we included programmatic child follow-up data in addition to maternal data. These included infants enrolled in care without corresponding mothers' postdelivery data $(n=73,9.8 \%) .572(76.7 \%)$ were identified in both census data and at least one programmatic data source (maternal post-delivery and child follow-up). 151 births ( $20.2 \%$ of total) were identified only in the programmatic data, while 23 (3.1\%) were only identified in the census (Fig. 3).

Consistency in birth outcome classification: Most births $(n=566)$ were categorized consistently as 'living' in both data sources, comprising 99.4\% (95\% Cl: 98.8\%, 100\%) of the 572 births identified in both sources and $76.3 \%$ (95\% Cl: $73.1 \%, 79.3 \%)$ of all 746 births (collectively from maternal and child records). In contrast, barring two infant deaths that were consistently identified in both methods, most were classified inconsistently in both sources, i.e. infant deaths in one source were classified as living or stillbirths in the other data source (Table 2), or were not recorded. Of note, programmatic sources identified five infant deaths and five stillbirths that were not recorded in the census data, compared to two infant deaths and one stillbirth that were recorded in census data but not in programmatic data. 
Table 2

Consistency in outcome classification for each birth identified in both census and programmatic data, $\mathrm{n}$ $=572$

\begin{tabular}{|llll|}
\hline & \multicolumn{3}{l|}{ Birth outcome classification in programmatic (routine) data } \\
\hline Birth outcome classification in census & Stillbirths & Deaths & Living \\
& $\mathbf{n}(\%)$ & $\mathbf{n}(\%)$ & $\mathbf{n}(\%)$ \\
\hline Stillbirths, $\mathbf{n}(\%)$ & $1(0.2 \%)$ & $0(0.0 \%)$ & $0(0.0 \%)$ \\
\hline Deaths, $\mathbf{n}(\%)$ & $1(0.2 \%)$ & $2(0.3 \%)$ & $1(0.2 \%)$ \\
\hline Living, $\mathbf{n}(\%)$ & $0(0.0 \%)$ & $1(0.2 \%)$ & $566(99 \%)$ \\
\hline
\end{tabular}

\section{Discussion}

We compared the consistency of data collected by $\mathrm{CHWs}$ on vital events that occurred during a one-year period using two different data collection methods. Programmatic data captured more births from maternal records than a census-based birth history (646 versus 593). Both approaches seemed to capture some unique births that the other did not -120 births were captured by the programmatic data alone, and 67 were captured by the census alone. Programmatic data also captured more birth records collectively from mothers and children than census data (723 versus 595). We observed very high consistency $(>95 \%)$ in classifying vital events among records identified in both methods. Although further research is needed to draw more inferential conclusions about data completion and quality, our findings seem to favor tracking vital events through $\mathrm{CHWs}$ ' routine household visits for program monitoring and evaluation, especially in a limited resource setting.

One of the reasons for fewer births identified in the census was that 43 women who consented to enroll in routine care through the broader RMNCH study did not agree to provide a birth history during the 2018 census. In this setting, the high rate of in- and out-migration poses challenges in care delivery, and may explain why some events were missing in the programmatic data $(25,26)$. This is consistent with other studies that have identified migration as a potential factor affecting vital events recording by $\mathrm{CHWs}(22)$. Future studies, especially using qualitative methods, can help explore why some events were missed by the programmatic data, and why women may have declined to provide birth histories during the census.

Although we observed high consistency in birth location classification (institutional and non-institutional) among births (from maternal records) identified in both sources (95.8\%), there was lower consistency in the sensitivity analysis (72\%, Supplementary Table 1$)$. However, since non-missing records from both sources tended to be classified as "institutional" births, it is likely that the missing records would also have been classified the same way, which would lead to higher consistency. Of note, we used broad categories and did not compare more granular birth locations within these categories (e.g. "hospital" 
versus "health post" among institutional births). Further, the high consistency in birth outcome classification (stillbirth, living, and death) for births (collectively from maternal and child records) identified in both methods was almost entirely driven by those in the much larger "living" category. Although both approaches missed some adverse infant outcomes that the other captured, programmatic data identified more deaths and stillbirths than the census. In retrospective data collection, it is common to either fail to report or inaccurately report past events over time (27). Recall bias may be one reason for missing events from the census method, among other potential reasons including declining participation, and age heaping $(28,29)$.

Similar studies in other low-resource settings that validated routine data collected by CHWs using different methods have shown varied findings (7). These studies were conducted as part of the "Real-time Monitoring of Under-Five Mortality" (RMM) project in different countries in Africa (14). In Mali, the team validated the routine data collected by lay volunteer community-based workers with household censusbased full birth history survey data collected by the same volunteer community-based workers. Their study spanned 20 villages with a catchment population of approximately 32,000 . Two full-time field coordinators conducted supervision, data verification, and data reviews for feedback loops to support community-based workers. The vital events data that $\mathrm{CHWs}$ reported were comparable with the census data and produced similar estimates of under-five mortality (30). In Malawi, the team compared the expected number of birth and death estimates obtained from routine data collected by health surveillance assistants (HSAs) with rigorous household surveys, collecting the complete birth history of women (aged 15-49) in approximately 24,000 households. Two different studies were conducted in two phases at different times, with enhanced supervision and data quality management in the second phase. Each HSA had a supervisor at a health center, who was responsible for field assessment and data quality reviews $(22,31)$. HSAs severely underreported births and deaths in both phases despite increased supervision and data quality in the second phase. On average, HSAs underreported births by $44 \%$ and under-five deaths by $49 \%$ over the study period. Joos et al. (2016) cited the challenges of the existing government health systems and high turnover rates of HSAs as the potential reasons for poor data quality (31). In Ethiopia, a validation study was conducted in two rural zones covering a total population of about 4.4 million. The team compared the vital events data collected by a professionalized home-visiting cadre, health extension workers (HEWs), with the data from a household mortality survey. The household mortality survey data were collected using a stratified two-stage cluster sampling design, as part of a larger evaluation that reached approximately 28,000 households. This validation study found severe underreporting of vital events when compared to household survey estimates - HEWs only reported $30 \%$ of births and $21 \%$ of under-five births causing underestimation in mortality rates. The researchers mentioned the high workload and challenges of supervision in remote areas as some of the potential reasons for low-quality data reported by HEWs (3).

Our findings were more consistent with the Mali study, where CHWs were able to identify the majority of vital events equally from both approaches. The approaches that the Mali study and our study used were also similar - both were smaller scale studies comparing routine data with census-based birth history data collected by CHWs. CHWs in Mali were also able to identify more events from the routine data 
collection method than the census method, which was similar to our findings (30). While CHWs in all the studies received some level of supervision and training for data collection, their incentives varied by setting. CHWs in our setting and Ethiopia were salaried, whereas those in Malawi and Mali received some incentives for data collection $(3,22,30,31)$. However, salaried HEWs in Ethiopia were not able to report complete and quality data due to other local challenges (3). These findings suggest that a combination of different factors can impact the quality of data reported by $\mathrm{CHWs}$. One key difference between our study and these other studies was our use of a mobile platform instead of paper-based tools or registers. Although CHWs in Ethiopia were part of a salaried and professionalized cadre like those in our study, they used paper-based tools, and research assistants later entered data into the database manually (3). In contrast, $\mathrm{CHWs}$ in our study used a mobile platform with built-in data validation, thereby eliminating incomplete form submissions and the need for manual data entry. In our experience, this was more efficient and less resource-intensive in ensuring better data quality. However, further studies are needed to investigate the complex factors that affect the quality and reliability of $\mathrm{CHW}$ routine data collection.

Differences in data quality assurance processes could have contributed to some of the inconsistencies we observed between the two data sources in our study. We began implementing regular data quality checks at the beginning of the census in 2018. Since programmatic data collection preceded the retrospective census data collection and extensive data quality checks, programmatic data quality might have been affected to some extent. However, there were some built-in data validations in the CommCare forms to reduce anticipated errors during programmatic data collection. Furthermore, since household data were being updated during the census, we found that this introduced some discrepancies in records, such as both old and new household IDs being retained when merging data during analysis. However, this seemed to be the case for only a few records.

There are several limitations to our study. One key limitation was the small study site comprising 14 wards. Infant death is a rare event, and the small numbers we observed limit our ability to make robust inferences using mortality data. Factors such as differences in $\mathrm{CHWs}$ ' length of employment, educational level, other competing work priorities, and training may also affect individual data collection. Since the same $\mathrm{CHW}$ collected data for her ward using both methodologies in this study, we likely mitigated the effects of these factors when comparing the two approaches. However, this can be a limitation as well: since census data collection followed programmatic data collection, $\mathrm{CHWs}$ may have retrieved memories from their care delivery to the same women, which could have influenced the data reported in the census. As has been noted in other studies, CHWs may have experienced a potential conflict of interest since they work to improve health outcomes in their communities, and are also asked to report data on adverse outcomes such as infant deaths (22). This seems unlikely in our study, however, as the census data also captured fewer births (in addition to fewer unfavorable outcomes) compared to the programmatic data.

In our experience, conducting the census in addition to CHWs' existing workload was resource- and timeintensive. Although pregnancy screening was temporarily halted during the census, CHWs continued to deliver and collect data for services such as antenatal, postnatal, and early childhood care. Census data collection took longer than the expected four months to complete, and the community may have

Page $12 / 20$ 
experienced survey fatigue. Frequent migration in the setting also posed challenges to completing census data collection within a shorter time period.

Our methods also lacked rigor in collecting mortality and stillbirth data. Although many LMICs commonly use a birth history method for mortality data, it is not always reliable for collecting information on stillbirths and neonatal deaths. There is a high chance of misclassification of self-reported stillbirths and neonatal deaths with this approach (32). Additionally, stillbirths and miscarriages may often be tied to religious and cultural beliefs in these communities (33). Thus, women may not openly disclose such events in a birth history. This could have caused misclassification or under-reporting of mortality and stillbirths in the programmatic data as well. However, this limitation may have been partially mitigated as $\mathrm{CHWs}$ belong to the same community as the women they serve, and have built trust with them through continued engagement during care delivery (34). Future studies should attempt to use a more advanced and in-depth method, such as verbal and social autopsy and participatory analytic methods, and strengthen linkages with government reporting systems to identify stillbirth and mortality events with greater accuracy $(8,13)$.

\section{Conclusions}

Salaried, trained and supervised community health workers are a promising cadre that can help address gaps in routine data collection while delivering care in low-resource settings. Despite some limitations, vital events recorded routinely by $\mathrm{CHWs}$ while delivering an integrated $\mathrm{RMNCH}$ intervention in rural Nepal were comparable to those collected using a birth history census. Our findings suggest that, despite some limitations, data from routinely tracking pregnancies, births, and deaths over time are promising and seem reliable for timely program monitoring and evaluation at a small scale, and may even be more effective than asking women to recall these events. These findings may offer insights to other lowresource settings aiming to use $\mathrm{CHW}$-collected data for timely tracking of progress of health outcomes, in the absence of a comprehensive vital registration system.

\section{List Of Abbreviations}

CHNs = Community Health Nurses

CHWs $=$ Community Health Workers

DHS= Demographic and Health Surveys

LMICs= low- and middle-income countries

$\mathrm{NHN}=$ Nyaya Health Nepal

$\mathrm{RMNCH}=$ reproductive, maternal, newborn, and child health 


\section{Declarations}

\section{Ethics approval and consent to participate}

The Nepal Health Research Council (461/2016), the Brigham and Women's Hospital (2017P000709/PHS), and the Icahn School of Medicine at Mount Sinai: (MSSM IRB-18-01091) institutional review boards approved this study for human subjects' research. Informed verbal consent was obtained at the beginning of enrollment into the larger $\mathrm{RMNCH}$ study. $\mathrm{CHWs}$ regularly received training on ethics, accompanied by close supervision from $\mathrm{CHNs}$ throughout the intervention, and participated in a specific refresher training session prior to the census in 2018. During the census, CHWs asked each participant for permission to proceed with asking about their birth history.

\section{Consent for publication}

Not applicable

\section{Availability of data and materials}

The datasets generated and/or analyzed during the current study will be posted in de-identified format in a publicly-accessible data repository, and are available from the corresponding author on reasonable request.

\section{Competing interests}

A. nTiwari, SS, and A. Thapa are employed by a US-based nonprofit (Possible). VB, LB, BB, SK, NM, HJR, and SP are employed by a nonprofit healthcare company (Nyaya Health Nepal, with support from the USbased nonprofit, Possible) that delivers free healthcare in rural Nepal using funds from the Government of Nepal and other public, philanthropic, and private foundation sources. NC, SH, DM, SM are employed by, and SM, DC, DM, and SS are faculty members at a private medical school (Icahn School of Medicine at Mount Sinai). DC is a faculty member at, DC and SH are employed part-time by, and SH is a graduate student at a public university (University of Washington). DM is a member on Possible's Board of Directors, for which he receives no compensation. RS is employed at an academic medical center (Brigham and Women's Hospital) that receives public sector research funding, as well as revenue through private sector fee-for-service medical transactions and private foundation grants. RS is a faculty member at a private medical school (Harvard Medical School) and employed at an academic medical center (Massachusetts General Hospital) that receives public sector research funding, as well as revenue through private sector fee-for-service medical transactions and private foundation grants. All authors declare that we have no competing financial interests. The authors do, however, believe strongly that healthcare is a public good, not a private commodity.

\section{Funding}


This study was conducted as part of a larger research study funded by the United States Agency for International Development via a Partnerships for Enhanced Engagement in Research award (sponsor grant number AID-OAA-A-11-00012, National Academy of Science sub award letter 2000007780). Dr. Duncan Maru received support from the Office of the Director, National Institutes of Health under an Early Independence Award, number DP50D019894. The Eunice Kennedy Shriver National Institute of Child Health \& Human Development (NICHD) and the National Institute of Dental \& Craniofacial Research (NIDCR) provided support for this award. The funders played no role in research design, data collection, data analysis, manuscript write-up, or decision to publish. Any opinions, findings, conclusions, or recommendations expressed in this article are those of the authors alone and do not necessarily reflect the views of the United States Agency for International Development or the National Academy of Science.

\section{Authors' contributions}

SM, DM, NC, and A. Thapa led the conception and design of the study with statistical advice from LS. The study grew out of discussion between SM, NC, A. Tiwari, WW, SP, DC, DM, SH, SS, RS, and A. Thapa, BB, HJR, and SK helped implement the intervention. A. Tiwari, VB, LB, NC, SP, and NM extracted, cleaned, and managed study data to maintain research data integrity. NC and A. Tiwari conducted analyses with input from LS, SM, DM, WW, and SS. A. Tiwari, NC, and SM drafted the manuscript with input from all authors. MB provided expert feedback for the manuscript. All authors contributed technical guidance to the intervention, and further drafted, reviewed, and approved the final manuscript.

\section{Acknowledgements}

We wish to express our appreciation to the Nepal Ministry of Health and Population for their continued efforts to improve the public-sector healthcare system in rural Nepal. Lastly, we are deeply indebted to the community and hospital staff, whose commitment to serving patients and dedication to improving the quality of healthcare in rural Nepal continues to inspire us.

\section{References}

1. Corsi DJ, Neuman M, Finlay JE, Subramanian S. Demographic and health surveys: a profile. International Journal of Epidemiology. 2012;41(6):1602-13.

2. UNICEF. Multiple Indicator Cluster Survey (MICS): UNICEF; [updated 2014 October 23. Available from: https://www.unicef.org/statistics/index_24302.html.

3. Amouzou A, Kidanu A, Taddesse N, Silva R, Hazel E, Bryce J, et al. Using Health Extension Workers for Monitoring Child Mortality in Real-Time: Validation against Household Survey Data in Rural Ethiopia. PLOS ONE. 2015;10(11):e0126909.

4. Aqil A, Lippeveld T, Hozumi D. PRISM framework: a paradigm shift for designing, strengthening and evaluating routine health information systems. Health Policy and Planning. 2009;24(3):217-28.

5. Bryce J, Arnold F, Blanc A, Hancioglu A, Newby H, Requejo J, et al. Measuring Coverage in $\mathrm{MNCH}$ : New Findings, New Strategies, and Recommendations for Action. PLOS Medicine. 
2013;10(5):e1001423.

6. Fottrell E, Byass P. Population survey sampling methods in a rural African setting: measuring mortality. Population Health Metrics. 2008;6(1):2.

7. Silva R, Amouzou A, Munos M, Marsh A, Hazel E, Victora C, et al. Can Community Health Workers Report Accurately on Births and Deaths? Results of Field Assessments in Ethiopia, Malawi and Mali. PLOS ONE. 2016;11(1):e0144662.

8. Nichols EK, Ragunanthan NW, Ragunanthan B, Gebrehiwet H, Kamara K. A systematic review of vital events tracking by community health agents. Global Health Action. 2019;12(1):1597452.

9. Tulenko K, Møgedal S, Afzal MM, Frymus D, Oshin A, Pate M, et al. Community health workers for universal health-care coverage: from fragmentation to synergy. Bull World Health Organ. 2013;91(11):847-52.

10. Kok MC, Dieleman M, Taegtmeyer M, Broerse JE, Kane SS, Ormel H, et al. Which intervention design factors influence performance of community health workers in low- and middle-income countries? A systematic review. Health Policy and Planning. 2014;30(9):1207-27.

11. Braun R, Catalani C, Wimbush J, Israelski D. Community Health Workers and Mobile Technology: A Systematic Review of the Literature. PLOS ONE. 2013;8(6):e65772.

12. Källander K, Tibenderana JK, Akpogheneta OJ, Strachan DL, Hill Z, ten Asbroek AHA, et al. Mobile Health (mHealth) Approaches and Lessons for Increased Performance and Retention of Community Health Workers in Low- and Middle-Income Countries: A Review. Journal of medical Internet research. 2013;15(1):e17.

13. Basera TJ, Schmitz K, Price J, Willcox M, Bosire EN, Ajuwon A, et al. Community surveillance and response to maternal and child deaths in low- and middle-income countries: A scoping review. PLOS ONE. 2021;16(3):e0248143.

14. Bryce J, Group RMMW. "Real-Time" Monitoring of Under-Five Mortality: A Vision Tempered by Reality. PLOS Medicine. 2016;13(1):e1001912.

15. Regeru RN, Chikaphupha K, Bruce Kumar M, Otiso L, Taegtmeyer M. 'Do you trust those data?'-a mixed-methods study assessing the quality of data reported by community health workers in Kenya and Malawi. Health Policy and Planning. 2020;35(3):334-45.

16. UNICEF. Every Child's Birth Right: Inequities and trends in birth registration. December 2013.

17. Joshi C, Torvaldsen S, Hodgson R, Hayen A. Factors associated with the use and quality of antenatal care in Nepal: a population-based study using the demographic and health survey data. BMC Pregnancy and Childbirth. 2014;14(1):94.

18. Khanal V, Adhikari M, Karkee R, Gavidia T. Factors associated with the utilisation of postnatal care services among the mothers of Nepal: analysis of Nepal Demographic and Health Survey 2011. BMC Women's Health. 2014;14(1):19.

19. Paudel D, Shrestha IB, Siebeck M, Rehfuess EA. Neonatal health in Nepal: analysis of absolute and relative inequalities and impact of current efforts to reduce neonatal mortality. BMC Public Health. 2013;13(1):1239. 
20. Maru S, Nirola I, Thapa A, Thapa P, Kunwar L, Wu W-J, et al. An integrated community health worker intervention in rural Nepal: a type 2 hybrid effectiveness-implementation study protocol. Implementation Science. 2018;13(1):53.

21. Citrin D, Thapa P, Nirola I, Pandey S, Kunwar LB, Tenpa J, et al. Developing and deploying a community healthcare worker-driven, digitally- enabled integrated care system for municipalities in rural Nepal. Healthcare. 2018;6(3):197-204.

22. Amouzou A, Banda B, Kachaka W, Joos O, Kanyuka M, Hill K, et al. Monitoring Child Mortality through Community Health Worker Reporting of Births and Deaths in Malawi: Validation against a Household Mortality Survey. PLOS ONE. 2014;9(2):e88939.

23. Otieno CF, Kaseje D, Ochieng' BM, Githae MN. Reliability of Community Health Worker Collected Data for Planning and Policy in a Peri-Urban Area of Kisumu, Kenya. Journal of Community Health. 2012;37(1):48-53.

24. UNFCO. District Profile: Achham. UN Resident Coordinator and Humanitarian Coordinator Office; 2013.

25. Poertner E, Junginger M, Müller-Böker U. Migration in Far West Nepal. Critical Asian Studies. 2011;43(1):23-47.

26. Vaidya NK, Wu J. HIV epidemic in Far-Western Nepal: effect of seasonal labor migration to India. BMC Public Health. 2011;11(1):310.

27. Zimmerman LA, Shiferaw S, Seme A, Yi Y, Grove J, Mershon C-H, et al. Evaluating consistency of recall of maternal and newborn care complications and intervention coverage using PMA panel data in SNNPR, Ethiopia. PLOS ONE. 2019;14(5):e0216612.

28. Guillot M, Gerland P, Pelletier F, Saabneh A. Child Mortality Estimation: A Global Overview of Infant and Child Mortality Age Patterns in Light of New Empirical Data. PLOS Medicine. 2012;9(8):e1001299.

29. Espeut $D$, Becker $S$. The validity of birth and pregnancy histories in rural Bangladesh. Journal of Health, Population and Nutrition. 2015;33(1):17.

30. Munos MK, Koffı AK, Sangho H, Traoré MG, Diakité M, Silva R, et al. Strengthening Community Networks for Vital Event Reporting: Community-Based Reporting of Vital Events in Rural Mali. PLOS ONE. 2015;10(11):e0132164.

31. Joos O, Amouzou A, Silva R, Banda B, Park L, Bryce J, et al. Strengthening Community-Based Vital Events Reporting for Real-Time Monitoring of Under-Five Mortality: Lessons Learned from the Balaka and Salima Districts in Malawi. PLOS ONE. 2016;11(1):e0138406.

32. Liu L, Kalter HD, Chu Y, Kazmi N, Koffi AK, Amouzou A, et al. Understanding Misclassification between Neonatal Deaths and Stillbirths: Empirical Evidence from Malawi. PLOS ONE. 2016;11(12):e0168743.

33. Paudel M, Javanparast S, Dasvarma G, Newman L. Religio-cultural factors contributing to perinatal mortality and morbidity in mountain villages of Nepal: Implications for future healthcare provision. PLOS ONE. 2018;13(3):e0194328. 
34. Wu W-J, Tiwari A, Choudhury N, Basnett I, Bhatt R, Citrin D, et al. Community-based postpartum contraceptive counselling in rural Nepal: a mixed-methods evaluation. Sexual and Reproductive Health Matters. 2020;28(2):1765646.

\section{Figures}

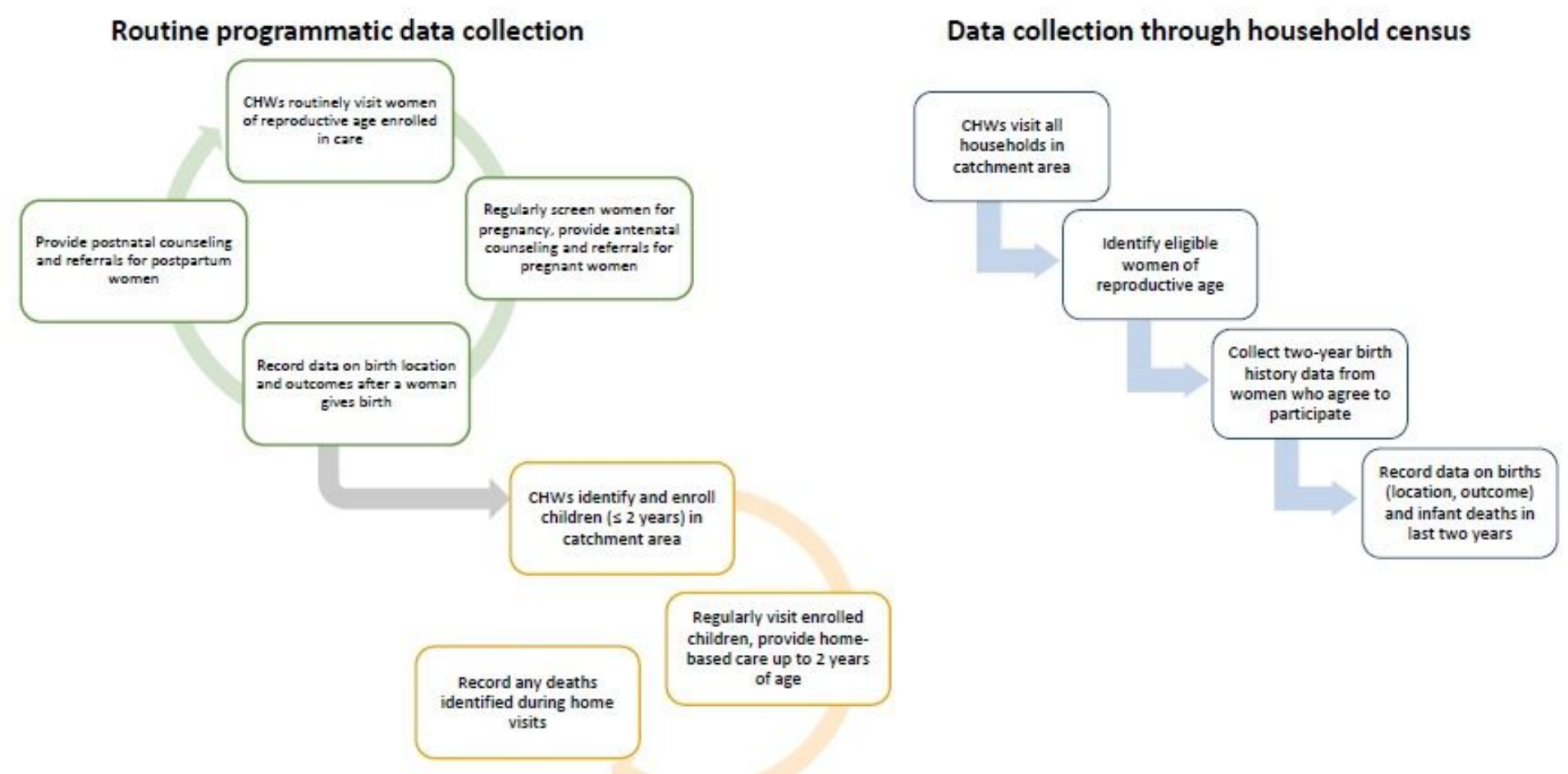

\section{Figure 1}

Summary of data collection methods 


\section{Births identified from maternal records $n(\%)$}

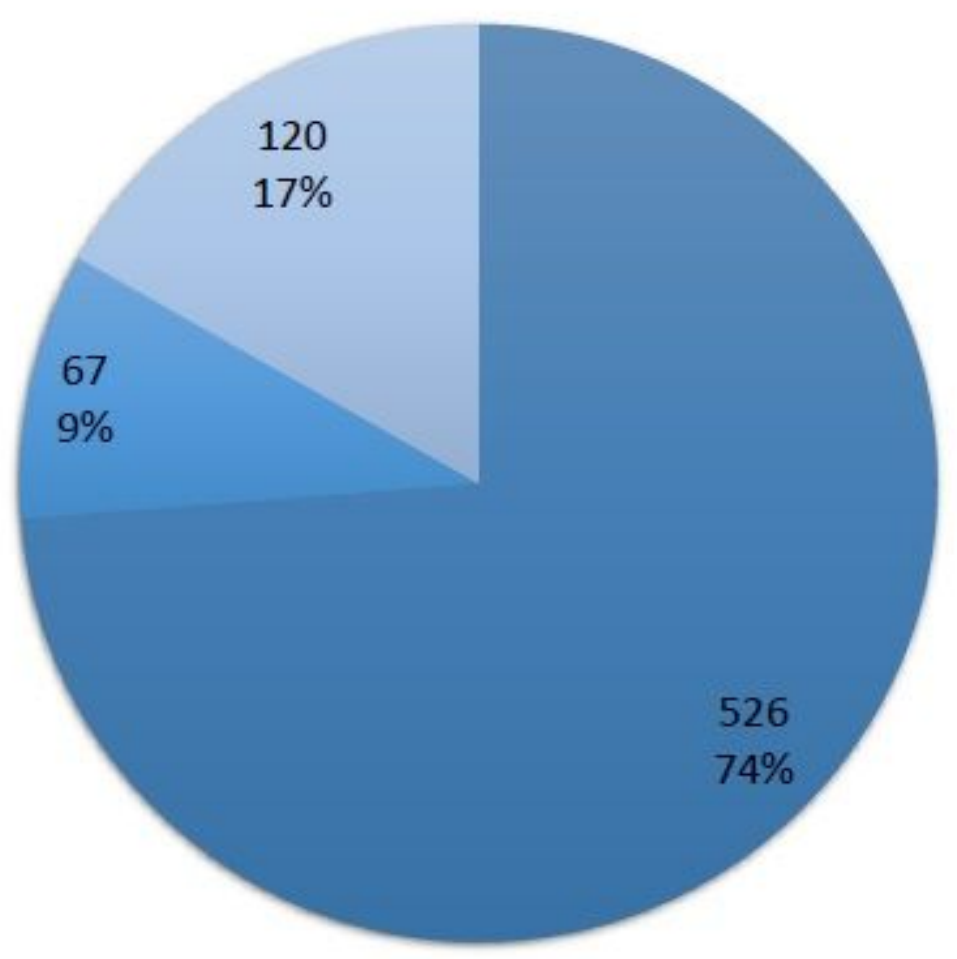

By both methods In census data alone In programmatic data alone

\section{Figure 2}

Summary of births identified (from maternal records) by census and programmatic data, $n=713$ 


\section{Births identified collectively from maternal and child records \\ $\mathrm{n}(\%)$}

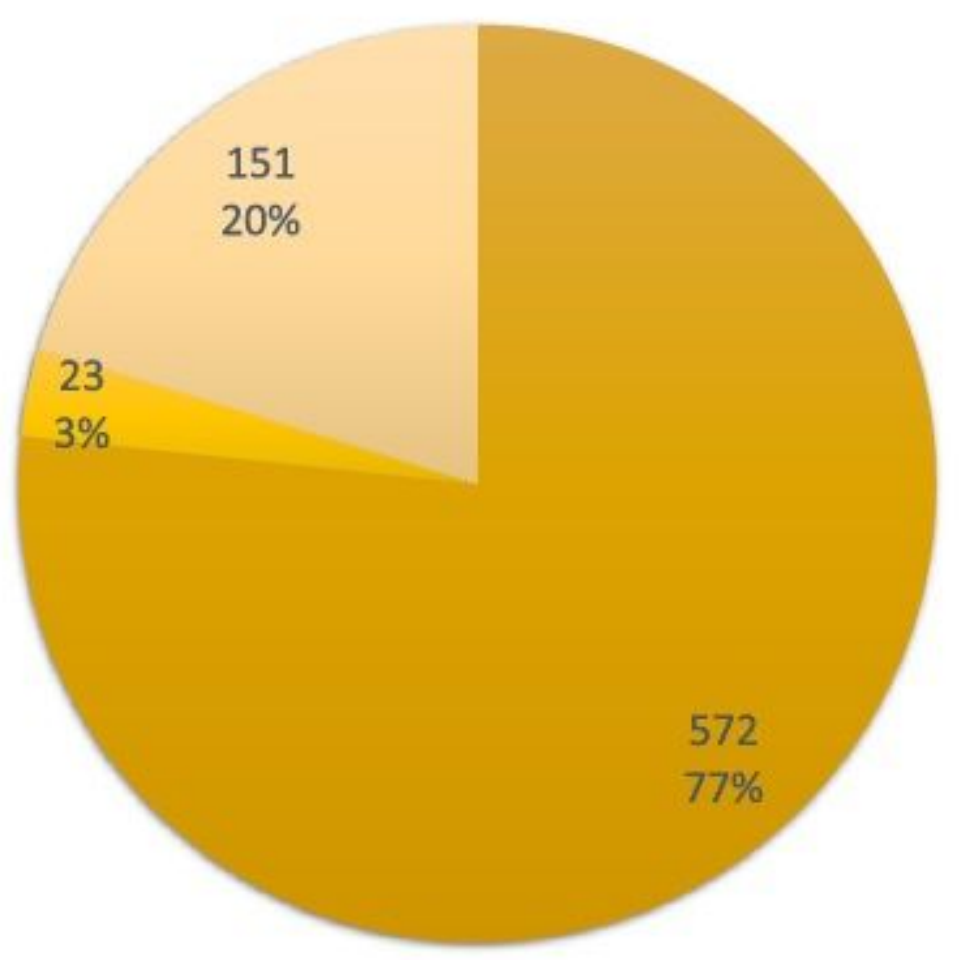

By both methods $\quad$ In census data alone In programmatic data alone

\section{Figure 3}

Summary of births (from maternal and child records) identified by census and programmatic data, $n=746$

\section{Supplementary Files}

This is a list of supplementary files associated with this preprint. Click to download.

- SupplementaryTable1.docx

- SupplementaryTable2.docx 